\title{
SEARCH FOR DIMENSIONALITY CROSSOVER OF SPIN-GLASS FREEZING IN SUPERLATTICES OF $\mathrm{Cd}_{0.50} \mathrm{Mn}_{0.50} \mathrm{Te} / \mathrm{CdTe}^{*}$
}

\author{
M. Sawicki, T. Dietl, G. Karczewsie, T. Wojtowicz, J. Kossut \\ AND T. SKośKIEWICZ \\ Institute of Physics, Polish Academy of Sciences \\ Al. Lotników 32/46, 02-668 Warszawa, Poland
}

SQUID measurements of the time decay of the thermoremanent magnetization (field-cooled in $1000 \mathrm{Oe}$ ) at long time scale, $10^{2}<t<10^{5} \mathrm{~s}$, are presented for MBE grown $\mathrm{Cd}_{0.50} \mathrm{Mn}_{0.50} \mathrm{Te}$. We found that for both thin $(32 \AA)$ and thick $(2500 \AA)$ layers the spin-glass dynamics is characterized by a similar value of $k=-\left(1 / T_{\mathrm{f}}\right)\left(\mathrm{d} T_{\mathrm{f}} / \mathrm{d} \log t\right)>0.05$, indicating the absence of the phase transition at nonzero temperature under the experimental conditions.

PACS numbers: 75.50.-y, 75.50.Rr, 75.50.Lk

Diluted magnetic semiconductors (DMS), such as $\mathrm{Cd}_{1-x} \mathrm{Mn}_{x} \mathrm{Te}$, are disordered IIeisenberg antiferromagnets in which both lattice favoured frustration and randomness may lead to a spin-glass-like state at low temperatures [1]. Recent progress in fabrication techniques, such as molecular beam epitaxy (MBE) [2], has made it possible to investigate properties of the spin-glass phase of DMS in the form of very thin layers [3], thus enabling us to address the problem of the lower critical dimensionality for this system [4]. To face this problem we investigated low temperature properties of $\mathrm{Cd}_{0.50} \mathrm{Mn}_{0.50} \mathrm{Te}$ layers with varying thicknesses: $5,10,15$, and 20 monolayers (MLs). In order to improve the signal-to-noise ratio, the samples were grown in the form of superlattices with about 200 periods, and with nonmagnetic CdTe spacers thick enough $(16 \mathrm{ML} \approx 50 \AA)$ to preclude the interlayer magnetic coupling. We found so far that the freezing temperature $T_{\mathbf{f}}$ does depend on the width of the magnetic layer $w$, namely it scales with $T$ as $T_{\mathrm{f}} \propto w^{0.8}$ [4], i.e. in the way theoretically expected for thin layered spin glasses measured over a long yet limited time scale [5].

Within the droplet-scaling theory the observation time $t$ is directly related to a length scale, $L(t)$, on which the dynamics is probed. Thus, as long as $L(t)$ is smaller than $w$ the spin-glass film displays 3D dynamic behaviour and crosses over

*Work supported by the grant 2 P03 B 06708 from the Committee for Scientific Research. 
to yield 2D dynamics in the opposite case. Therefore the measurement of the time dependent magnetization is expected to shed some light on the problem of the lower critical dimensionality in spin glasses with merely short range antiferomagnetic interactions. On the other hand, the dynamics of spin glasses on time scales longer than the maximum relaxation time of the system at given temperature is independent of frequency and is governed by the magnetic field [6].

Because of the cation exchange mechanism (interdiffusion), there is a vast number of weakly coupled or even loose Mn moments at the CdTe-CdMnTe interfaces, i.e., around the high- $x$ CdMnTe cores [7]. These moments give rise to a paramagnetic $(\propto 1 / T)$ component in the measured signal, which, particularly at low temperatures, obscures considerably the glassy behaviour of the magnetic layers. This makes it virtually impossible to track down accurately the spin-glass dynamics from the measurements performed in the external magnetic field. On the other hand, as it is known, a remanent magnetization is left on the sample when the external field is quenched at $T<T_{\mathrm{f}}[8]$. Thus, in the casir of a DMS superlattice the signal from loose Mn ions averages out after switching off the field, leaving the pure remanent magnetization stemming only from the core of the investigated magnetic layers. Therefore, the investigation of the remanent magnetization is our method of choice to get the most accurate information on spin-glass freezing for very thin layers of DMS.

We present the results of the measurements of the time decay of the thermoremanent magnetization (TRM) in a thin layer $\left(10 \mathrm{ML} \mathrm{Cd} \mathrm{Cd}_{0.50} \mathrm{Mn}_{0.50} \mathrm{Te} / \mathrm{CdTe}\right.$ superlattice, $w \approx 32 \AA)$ and in a thick epilayer $(w=25000 \AA)$ of $\mathrm{Cd}_{0.45} \mathrm{Mn}_{0.55} \mathrm{Te}$, also MBE grown, as a reference sample. Both samples have a similar quasi-static $\mathrm{T}_{\mathrm{f}}(\approx 10 \mathrm{~K}$ and $\approx 12 \mathrm{~K}$, respectively). A spin glass is subject to magnetic ageing at $T<T_{\mathrm{f}}$ [9], which manifests itself in a dependence of the time relaxation of the magnetization on the wait-time $t_{\mathrm{w}}$ at $T=$ const before the field is changed. It is therefore imperative to use a well-defined procedure when measuring relaxation phenomena. In this study, each sample was field-cooled from the equilibrium through $T_{\mathrm{f}}$ to the working temperature $T$ at the rate of about $1.5 \mathrm{~K} / \mathrm{min}$. In order to resolve the signal with an adequate resolution, the applied magnetic field was $H=1000$ Oe. Then, after $t_{\mathrm{w}}=300 \mathrm{~s}$ the field was quenched and the data acquisition commenced. A post-quench magnetic field instabilities within the superconducting coil did not allow us to collect good quality data for $t<100 \mathrm{~s}$. The measurement was performed until about $10^{5} \mathrm{~s}$, which extends by far the time range of previous studies of the dynamics in DMS [1,10-13]. Finally, the sample was heated up above $T_{\mathrm{f}}$, where the zero level of the signal was established. The procedure was repeated for several temperatures in the range $0.4 T_{\mathrm{f}}<T<T_{\mathrm{f}}$.

Figures $1 \mathrm{a}$ and $\mathrm{lb}$ show time dependence of TRM for both samples. Surprisingly, for both samples we observe essentially the same behaviour. At low $T$ almost $\log t$ dependence is observed with roughly the same relative relaxation rate ${ }^{\dagger}$. At temperatures approaching $\mathrm{T}_{\mathrm{f}}$ a more complex pattern develops. In this time domain, the time decay of the TRM should follow the stretched exponential de-

tThe ordinary relaxation rate is obviously higher at the superlattice sample, since as reported previously [4], the TRM accumulated in the superlattice is a few times higher than in bulk samples. This fact is not accounted for at present. 

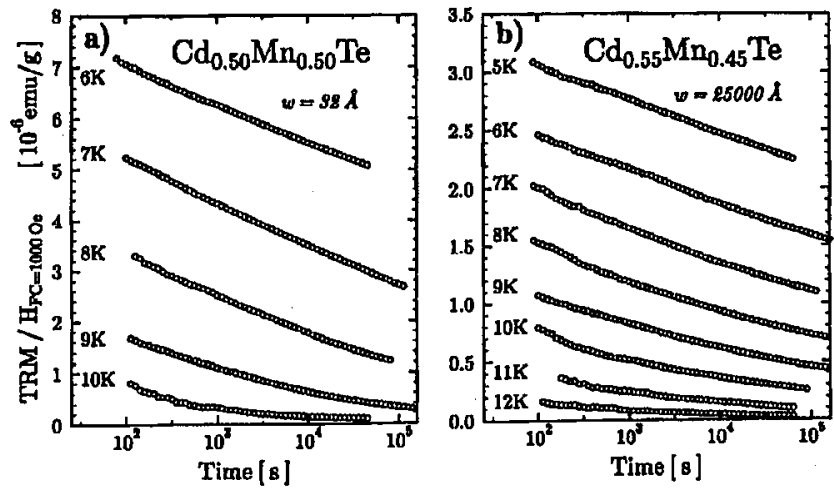

Fig. 1. Thermoremanent magnetization after $\mathrm{FC}$ at $1000 \mathrm{Oe}$ as a function of time in (a) $w=32 \AA$ superlattice and (b) $w=25000 \AA$ thick layer.
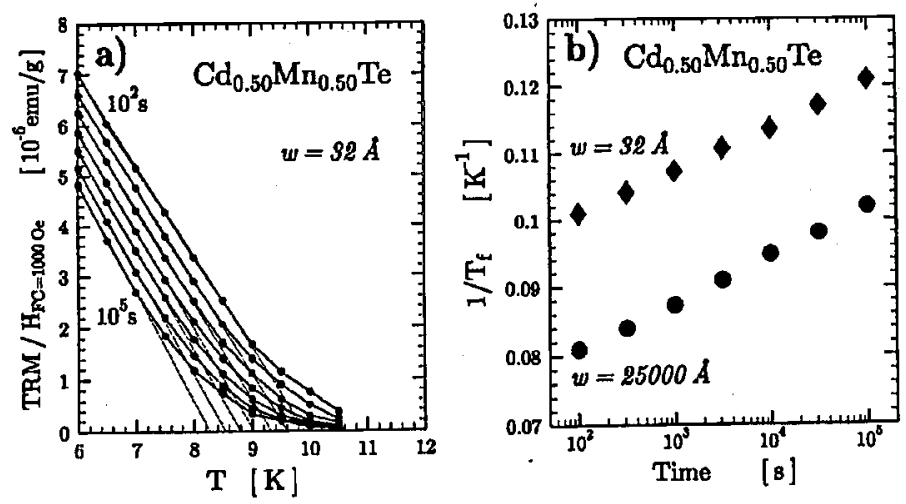

Fig. 2. (a) Thermoremanent magnetization as a function of temperature for constant observation time (an example for the superlatttice). (b) Inverse of the $T_{\mathrm{f}}$ as the function of time for both the superlattice (diamods) and thick layer (circles).

pendence: $M_{\mathrm{TRM}}(t)=M_{0} \exp \left[-\left(t / t_{0}\right)^{1-n}\right]$, with the time-stretch exponent $n$ close to 0.75 [14]. This however does not happen. For the thick layer the time-stretch exponent $n$ is 1 at all temperatures, so the power-law decay, $M_{\mathrm{TRM}}(t) \propto t^{-a}$, governs totally the relaxation. We get a very similar result for the superlattice, $n \approx 1$. In the latter, however, we observe that $n$ is also weakly time dependent at some temperatures, suggesting an admixture of a logarithmic decay. At present it is difficult to assign this difference to a size effect, since compositional imperfections of the superlattice would have resulted in a broader spectrum of relaxation times.

The data from both figures can be taken to plot the temperature dependence of TRM for different observation times. An example for the superlattice is shown in Fig. 2a. According to the principle of superposition of the time relaxation, the $T_{f}$ values for each observation time can be established by the linear extrapolation 
of $\operatorname{TRM}(T)$ dependence to zero, see the figure. Figure $2 \mathrm{~b}$ collects the value of $T_{\mathrm{f}}(t)$ established this way for both studied layers. We see that over the studied time domain $T_{\mathrm{f}}$ scales to zero as $\log t \rightarrow \infty$, thus indicating $T_{\mathrm{c}}=0$. This striking conclusion follows also from a relatively large value of $k=-\left(1 / T_{\mathrm{f}}\right)\left(d T_{\mathrm{f}} / d \log t\right)$, i.e. $k=0.055$ and $k=0.075$ for the superlattice and the thick layer, respectively. Such findings may indicate that both samples are below the critical dimensionality for the phase transition, which according to current theory of spin glasses is between 2 and $3[5,15]$. On the other hand, the observed proportionality $T_{\mathrm{f}}^{-1} \propto \log t$, see Fig. 2b, implies that the correlation length is independent of temperature at this constant field $[5,6]$. Therefore, we may deal with the case that for the investigated layers the scaling behaviour has been already affected by the external field of 1000 Oe.

We are presently extending our measurements for weaker magnetic fields, Bridgman grown bulk samples, and superlattices with other thicknesses of magnetic layers.

\section{References}

[1] C. Rigaux, Ann. Phys. (France), Coll. C2, Supp. n.3 20, 149 (1995) and references therein.

[2] N. Samarth, H. Luo, J.K. Furdyna, T.M. Giebultowicz, D.D. Awschalom, Acta Phys. Pol. A 82, 573 (1992).

[3] M. Sawicki, M.A. Brummell, P.A.J. de Groot, G.J. Tomka, D.E. Ashenford, B. Lunn, J. Cryst. Growth 138, 900 (1994).

[4] M. Sawicki, S. Koleśnik, T. Wojtowicz, G. Karczewski, E. Janik, M. Kutrowski, A. Zakrzewski, T. Dietl, J. Kossut, Superlattices Microstruct. 15, 475 (1994); M. Sawicki, T. Dietl, T. Skośkiewicz, G. Karczewski, T. Wojtowicz, J. Kossut, Acta Phys. Pol. A 88, 1038 (1995).

[5] D.A. Fisher, D. Huse, Phys. Rev. B 36, 8937 (1987); L. Hoines, R. Stubi, R. Loloee, J.A. Coven, J. Bass Phys. Rev. Lett. 66, 1224 (1991).

[6] J. Mattsson, T. Jonsson, P. Nordblad, H. Aruga Katori, A. Ito, Phys. Rev. Lett. 74, 4305 (1995).

[7] J.A. Gaj, W. Grieshaber, C. Bodin-Deshayes, J. Cibert, G. Feuillet, Y. Merle d'Aubigné, A. Wasiela, Phys. Rev. B 50, 5512 (1994).

[8] S. Oseroff, F.G. Gandra, J. Appl. Phys. 57, 3421 (1985).

[9] L. Lundgren, P. Nordblad, P. Svenlindh, O. Beckman, J. Appl. Phys. 57, 3371 (1985).

[10] M. Ayadi, J. Ferré, A. Mauger, R. Triboulet, Phys. Rev. Lett. 57, 1165 (1986).

[11] P. Nordblad, P. Svenlindh, J. Ferré, M. Ayadi, J. Magn. Magn. Mater. 59, 250 (1986).

[12] M. Smith, A. Dissanayake, H.X. Jiang, Phys. Rev. B 49, 4514 (1987).

[13] M. Dahl, A. Mergler, M. Sawicki, T. Litz, A. Waag, T. Gerhard, G. Karczewski, T. Wojtowicz, J. Kossut, J. Cryst. Growth 159, 1009 (1996).

[14] R.V. Chamberlin, G. Mazurkiewicz, R. Orbach, Phys. Rev. Lett. 52, 867 (1984).

[15] P. Granberg, P. Nordblad, P. Svenlindh, L. Lundgren, R. Stubi, G.G. Kenning, D.L. Leslie-Pelecky, J. Bass, J. Cowen, J. Appl. Phys. 67, 5252 (1990). 